\title{
Correction: YAP1 overexpression contributes to the development of enzalutamide resistance by induction of cancer stemness and lipid metabolism in prostate cancer
}

Hsiu-Chi Lee · Chien-Hui Ou • Yun-Chen Huang • Pei-Chi Hou • Chad J. Creighton (1) - Yi-Syuan Lin • Che-Yuan Hu • Shih-Chieh Lin (1)

Published online: 19 May 2021

(c) The Author(s), under exclusive licence to Springer Nature Limited 2021

Correction to: Oncogene

https://doi.org/10.1038/s41388-021-01718-4

Unfortunately, the institute name by affiliation 2 was given incorrect. The correct name is: Department of Urology,
National Cheng Kung University Hospital, College of Medicine, National Cheng Kung University, Tainan, Taiwan

Corresponding author of the paper is Shih-Chieh Lin Jaylin@mail.ncku.edu.tw

The original article has been corrected. 